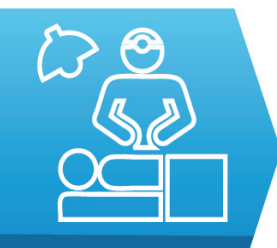

SURGERY

\title{
Intraoperative study of the functional perforasome - an experimental model
}

Maria-Eliza Nedu, Alexandru Dan Valentin Georgescu

Department of Plastic Surgery, Iuliu Hatieganu University of Medicine and Pharmacy, Cluj-Napoca, Romania
DOI: $10.15386 / \mathrm{mpr}-1896$

Manuscript received: 12.09.2020

Received in revised form: 16.01.2021

Accepted: 03.02.2021

Address for correspondence:

Nedu.Maria@umfcluj.ro

This work is licensed under a Creative Commons Attribution-NonCommercialNoDerivatives 4.0 International License

\begin{abstract}
Background and aims. The aim of this study is to find the most suitable protocol based on an animal experimental model, through the use of a fluorescent dye, determining also its minimal concentration needed to stain the skin after arterial injection, in order to evidence the functional perforasome by visual examination.

Methods. Methylene blue solution was used in order to determine the territory vascularized by one perforator on fresh cadavers in many studies which introduced, as a final result, the concept of perforasome. One of the most frequent complications of perforator flaps is partial flap necrosis which could be avoided by correctly assessing pre-operatively the functional perforasome surface.

Two groups of seven rats were used in order to establish a proper surgical protocol to evaluate the functional perforasome in vivo by injecting the dye. Also, the minimal concentration for methylene blue was experimentally determined.

Results. The direct injection into the femoral artery of the proper concentration of dye, $1 \mathrm{mM}$ for methylene blue and the clamping of all the branches except the medial branch of the superficial epigastric artery is a reliable model to study the functional perforasome.

Conclusions. Our study demonstrates that the intraoperative assessment with fluorescent dye of the functional perforasome by intra-arterial injection of methylene blue is an easy, affordable and very efficient method to reduce the number of partial necrosis of the perforator flaps.
\end{abstract}

Keywords: functional perforasome, direct perforator, experimental study, epigastric artery, methylene blue

\section{Introduction}

Described for the first time in 1988 by Kroll and Rosenfield in a series of cases in which the flaps were used for low posterior midline defects [1] and one year later by Koshima and Soeda based on the inferior epigastric artery [2], perforator flaps brought to the local random flaps the advantage of the island pedicled flap, which gives more rotational and translational freedom. Furthermore, having their origin in fascia-cutaneous flaps [3], they bring the advantages of the mentioned flaps without the disadvantage of donor site morbidity, by eliminating the need to use the fascia, preserving the muscle function and observing the main vascular pedicles [4].

One particular type of perforator flap is the propeller flap, which sums all the advantages mentioned above, also adding the possibility of primary donor site closure [5].

Although there are more types of perforator flaps, like pedicle perforator flaps, perforator-based flaps, perforatorplus flaps, and even free perforator flaps, all have the same physiology principle - perforasomes vascularized by one perforator, which improves the flap survival [6].

The concept of angiosome was not sufficient in order to describe the perforator's own unique vascular arterial 
territory $[7,8]$. That's why Saint-Cyr et al. [9] introduced the concept of "perforasome". An accurate description of a single perforator's vascular distribution and flow characteristics reduces the main drawback of perforator flap namely partial/total flap necrosis.

In order to prevent partial flap necrosis it is important to study not only the perforator axis but also the direct and indirect vessels which link the perforasomes. Those vessels expand the anatomic territory supplied by the perforator enlarging the skin territory which can be raised on a single perforator depending on the dynamic of choke vessels [10].

Casal et al. studied the blood supply to the integument of the rat abdomen demonstrating that the caudal half is irrigated mainly by the superficial caudal epigastric artery (SIEA) and vein (SIEV) and allowed them to graphically represent its angiosomes [11].

The aim of this study was to find the most suitable protocol on animal experimental models using florescent dye and to determine its minimal concentration needed to stain the skin after arterial injection, in order to evidence the functional perforasome by visual examination. The selected dye was methylene blue due to its antiinflammatory, antioxidant, antimicrobial properties described previously [12].

\section{Methods}

\section{Experimental animals}

The study was conducted in the animal research laboratory of our University of Medicine and Pharmacy, Cluj-Napoca, Romania, having the ethical committee authorization No. 199/07.02.2020. Fourteen male Wistar rats, weighing between $400-500 \mathrm{mg}$, were used for the study.

All the animals were housed under standard environmental conditions, in rat cages and offered standard rat chow and tap water. They were housed individually in the animal facilities of the institution under controlled temperature, humidity and photo periods. Prophylactic antibiotics were not used. The rats were distributed in two groups of 7 subjects, where each concentration of methylene blue was tested on 2 animals having in each group one rat injected with saline solution.

\section{Anesthesia}

All animals were anesthetized with Ketamine and Xylazine 2\% 2:1, intramuscular in the contralateral hip. The dose used was $12 \mathrm{UI} / 100 \mathrm{~g}$ mixture.

\section{Surgical procedure}

The rats were weighed in order to determine the precise amount of anesthetic needed. The surfaces of the operating desk were disinfected with alcoholic solution. All the surgical instruments were sterilized (in autoclave) before the procedure. Microsurgical and conventional instruments were used. The surgical microscope was prepared for the procedure.
All the procedures were performed using scrub cap, mask and sterile surgical gown.

An assistant helped with the anesthetic administration. The rat was placed in its cage until the anesthesia is established, evaluating its depth by toe pinching and observance of respiration rate throughout the entire procedure. When the anesthesia was installed, the hair over the ventral surface of the abdomen and inguinal area was removed by shaving with an electrical apparatus. The surgical field was disinfected using an alcoholic solution with 2 minutes of contact time before surgery. The entire surgical procedure was performed wearing sterile gloves.

The rat was positioned on the surgical table lying on the back and paws immobilized. Using a 15-blade scalpel, the skin was incised in a convex shape, taking into consideration the lower edge of the superficial epigastric angiosome (Figure 1).

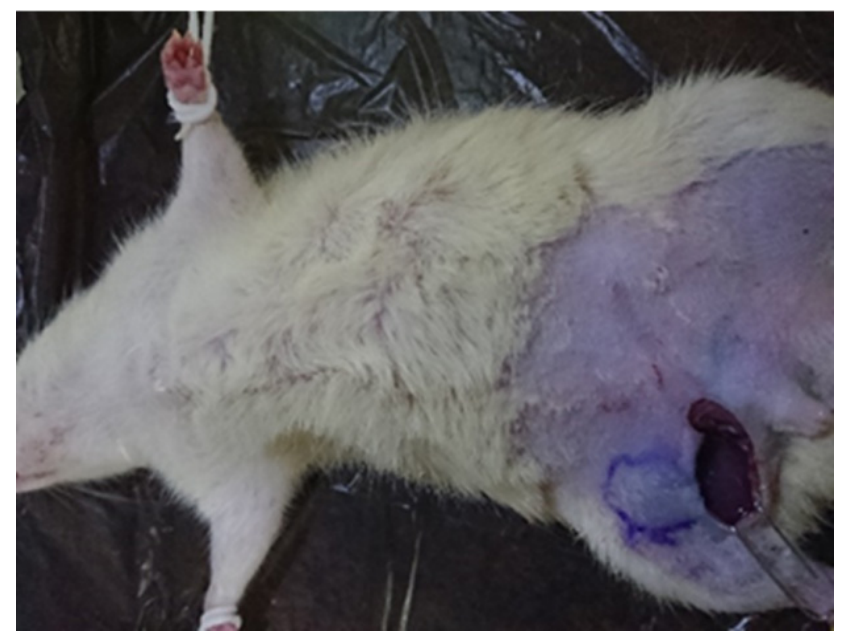

Figure 1. Skin incision on the lower edge of the superficial epigastric angiosome.
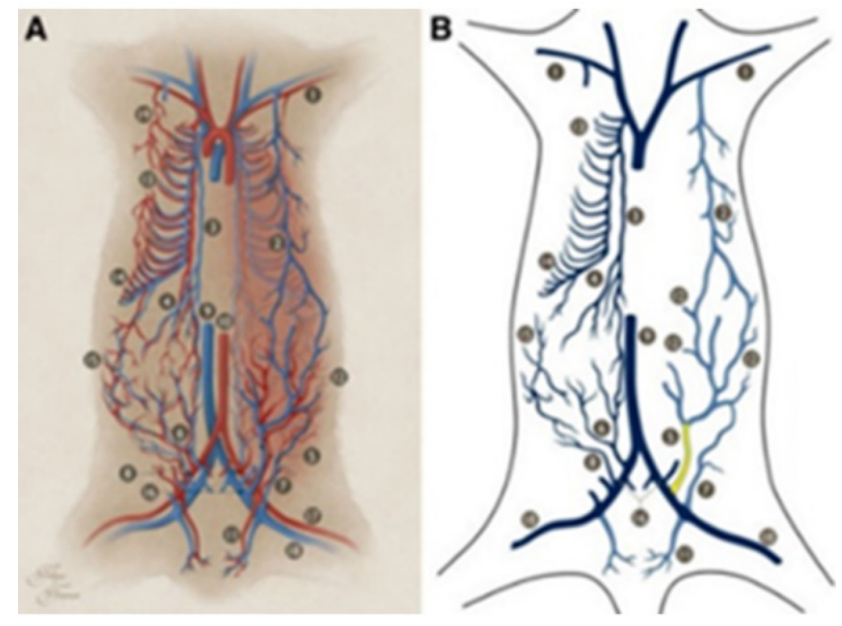

Figure 2. Macrovascular blood supply to the integument over the ventrolateral aspect of the abdomen of the rat [11]. 
The incision on the right side of the rat was about $5 \mathrm{~cm}$ length (Figure 1), to enable the dissection of the femoral vessels and its branches (Figure 2). After the incision was completed, the panniculus cavernous layer was reached. Then the dissection is continued until the muscular fascia was reached. Two retractors were used on the inguinal ligament site, one on the direction of the femoral artery and the other one on the direction of the homo lateral anterior paw. The dissection continued with the femoral vein and artery distal to inguinal ligament until $1.5 \mathrm{~cm}$ after the superficial epigastric artery origins. All the femoral artery branches were dissected for 0.5-1 $\mathrm{cm}$. The superficial epigastric artery was dissected until it split into the two branches, as presented in figure 3 and described [13].

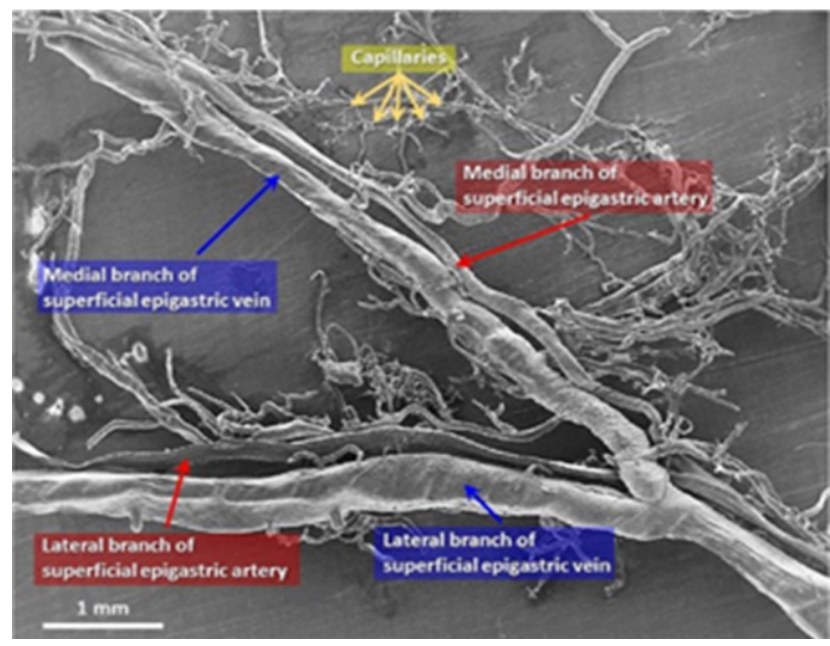

Figure 3. The bifurcation of the superficial epigastric artery into two branches [13].

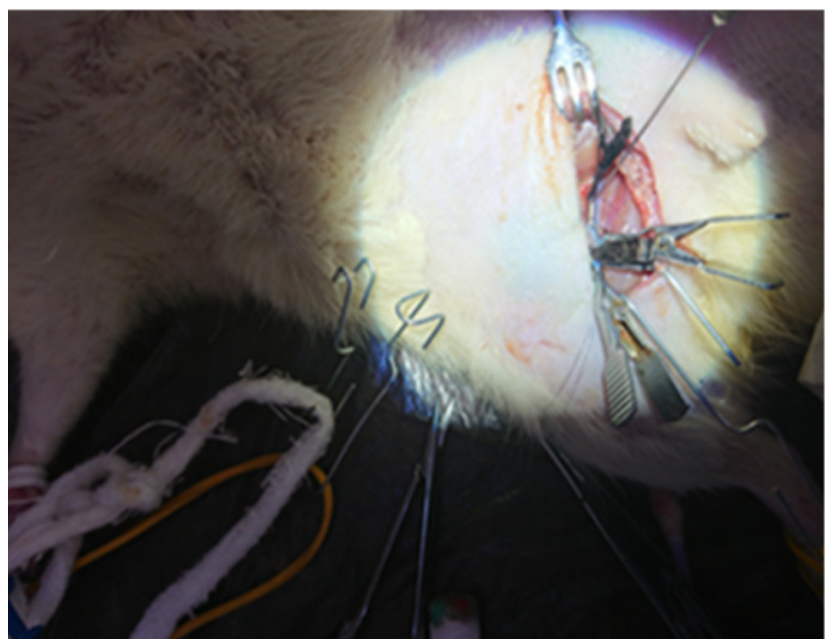

Figure 4. The femoral artery and its branches clamped except medial branch of superficial epigastric artery. Femoral artery incanulated.
The next step consisted of placing the clamps as follows: first clamp was placed on the femoral artery immediately after the inguinal ligament recording the clamping time. The second clamp was placed at $0.5 \mathrm{~cm}$ distal of the superficial epigastric artery origin continuing clamping the lateral femoral circumflex artery, branches for the adjacent muscles, saphenous and the lateral branch of the superficial epigastric artery. The vessels were washed with lignocaine. A small incision was made with a microsurgical scissor and a $29 \mathrm{G}$ catheter was introduced in the femoral artery distal to the inguinal ligament respectively proximal to the origin of the superficial epigastric artery (Figure 4). The catheter was fixed on the artery and its lumen is flushed with heparin.

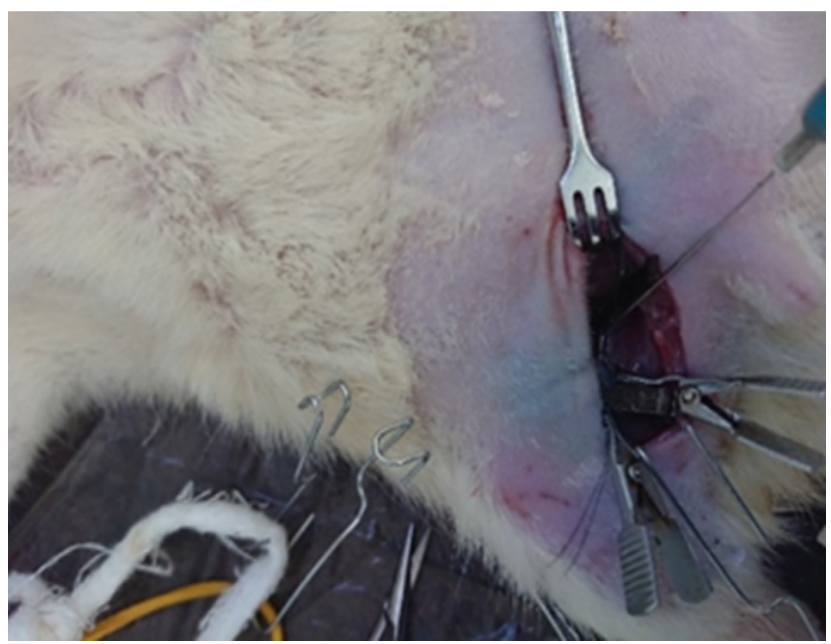

Figure 5. The skin coloration after dye injection.

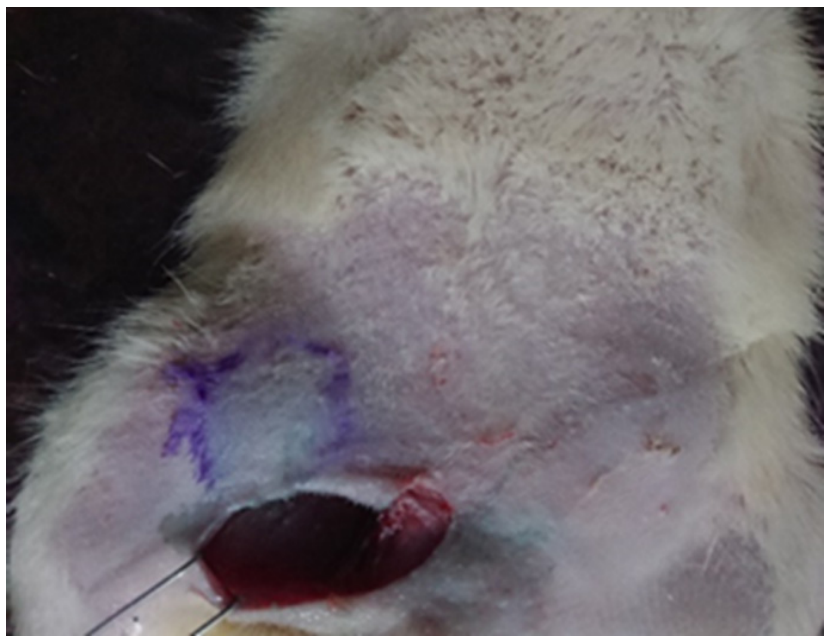

Figure 6. The marked margins (borders) of the functional perforasome identified by dye injection. 


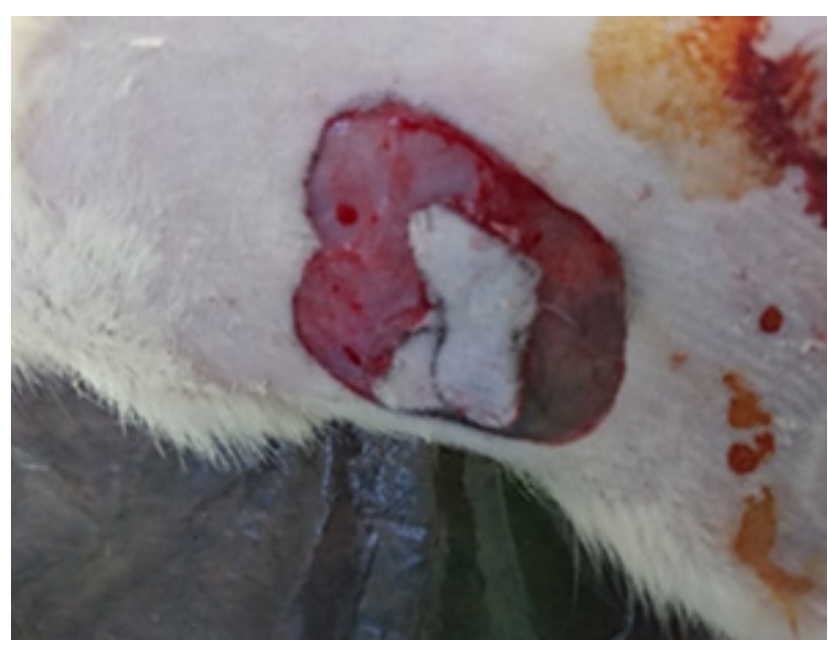

Figure 7. Flap elevation by incising the margins of the functional perforasome.

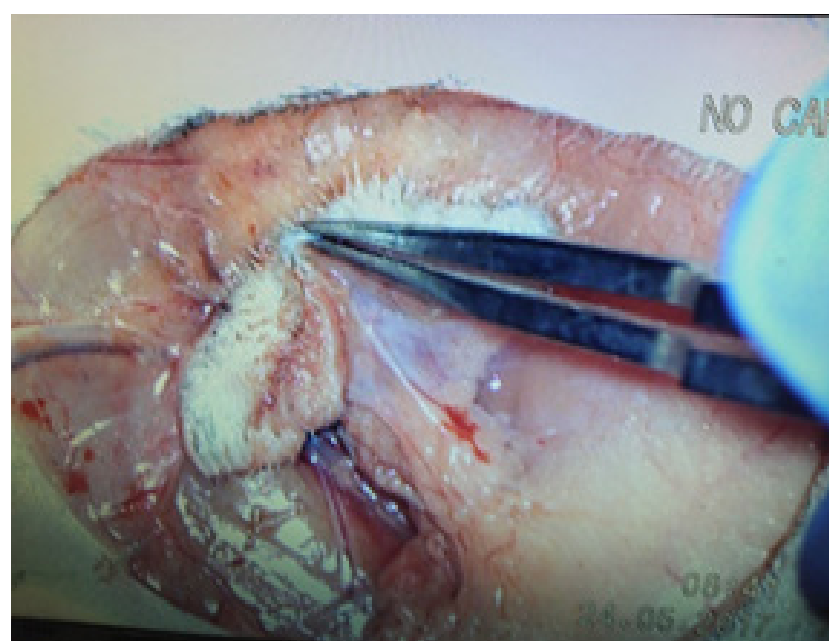

Figure 8. The direct perforator identified stained blue.

The methylene blue solution of dye was injected, and the skin was visually inspected to identify the coloured area (Figure 5). The margins of the area were drawn with a non-toxic marker (Figure 6). Then the lumen was washed again with the heparin-normal saline solution until no blood or debris were seen inside the vessels. The catheter was retracted, and the arterial opening was closed with 8.0 suture. All the clamps were removed.

An incision was made on the marks and the skin island was designed (Figure 7). The subcutaneous layer was dissected and the direct perforator was identified and isolated (Figure 8). The flap was raised and the initial incision was closed with 5.0 suture. The island was reattached on its place with continuous suture 5.0.

\section{Postoperative care}

The rat was left to recover inside its individual cage in left lateral decubitus position. The animal was monitored continuously turning it on the opposite lateral decubitus every 5 minutes, until it resumed sternal recumbency and was able to ambulate (Figure 9).

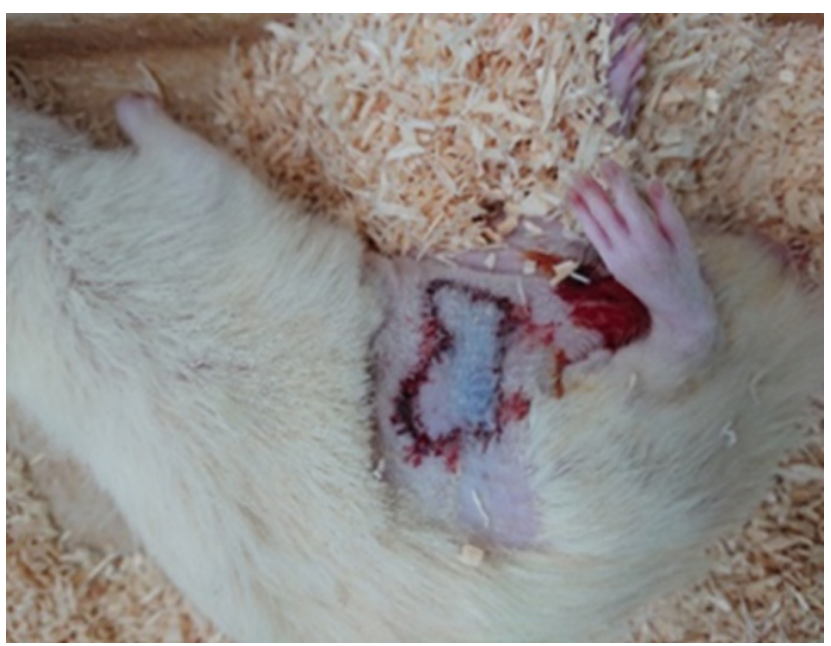

Figure 9. Left lateral decubitus position of the rat trying to return to sternal recumbency.

\section{Flap assessment}

The two groups of rats were monitored postoperatively and their health status was assessed. The first group was monitored daily for 5 days starting with first postoperative day and at the end of the second week (Figure 10). At the end of this period the rats were euthanized and the skin island harvested for histopathological exam.

The flaps were evaluated by visual inspection, presenting a food treat over the head of the rat. If exposure was insufficient, an assistant helped by changing the position of the rat during the flap examination. By the use of digital photography, the evolution was checked in order to evaluate the areas of wound dehiscence, flap epidermolysis, hyperaemia, congestion and necrosis (Figure 11).

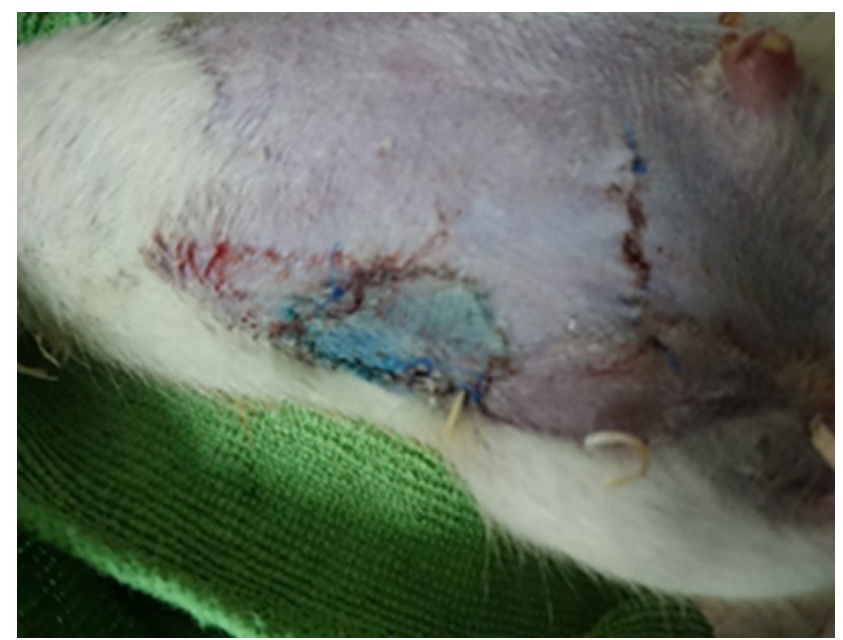

Figure 10. The flap in first postoperative day. 


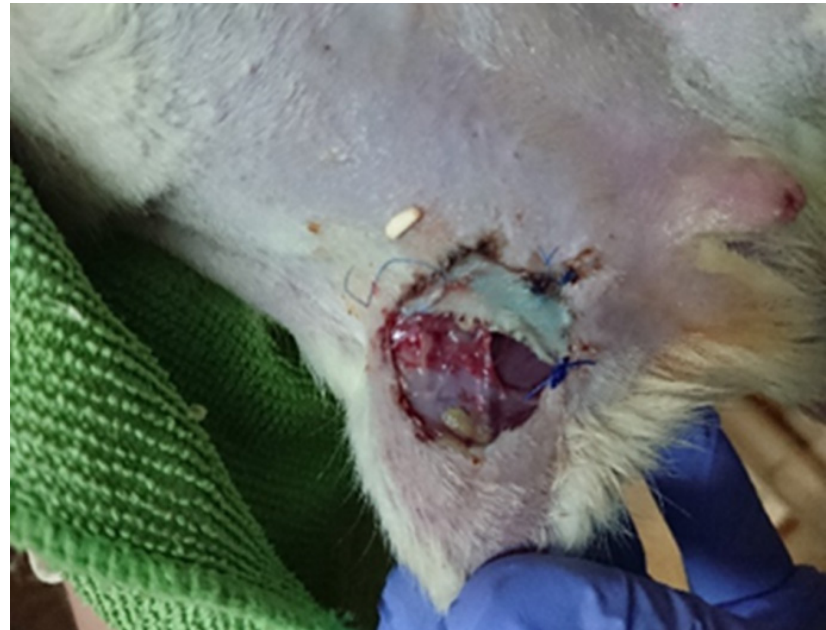

Figure 11. Dehiscence of the flap second day postoperative.

\section{Concentrations}

From the florescent dye (methylene blue), serum solutions were prepared with serum $0.9 \%$ (B. Braun Melsungen AG Germany) with concentrations between 0.5-5 $\mathrm{mM}$ for methylene blue.

In order to establish the minimal dye solution concentration, we injected the dye from the lowest to the highest concentration to determine the minimal concentration needed to colour the skin.

\section{Results}

Placing the incision on the inferior edge of the medial superficial epigastric artery angiosome as Casal et al. describes in their studies, as shown in figure 12, wound dehiscence and local necrosis is avoided [11].

By dissecting the branches of the superficial epigastric artery before injecting the dye, a smaller quantity of only $1 \mathrm{ml}$ of dye saline solution with a concentration of $1 \mathrm{mM}$ of methylene blue is needed to enable the accurate identification of the functional perforasome of a direct perforator.

The medial branch of superficial epigastric artery was closer to the incision having a direct vascular trajectory and thus minimizing the quantity of dye injected. Also, by clamping the rest of the femoral artery branches and superficial epigastric artery the quantity of dye absorbed by tissues was significantly reduced.

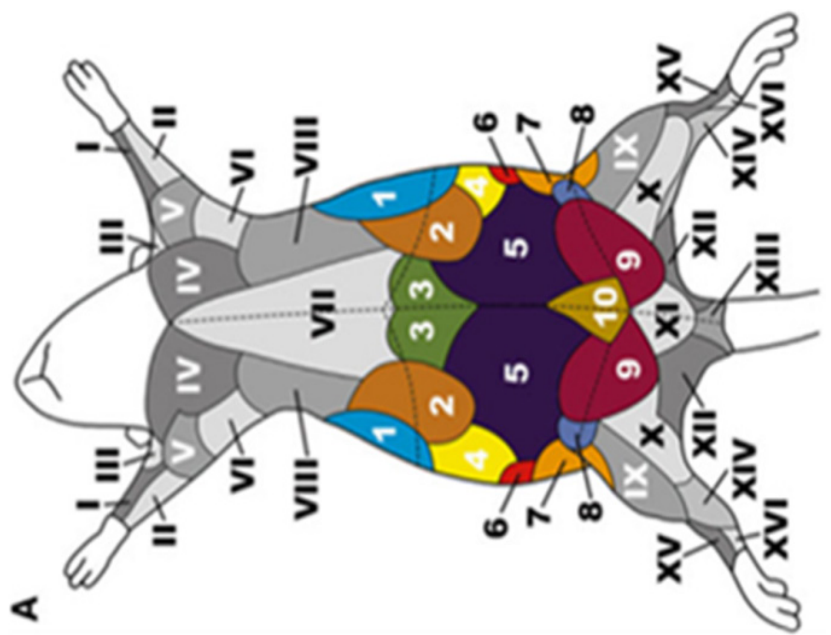

Figure 12. Schematic representation of the angiosomes of the ventrolateral aspect of the rat. The Arabic numbers represent the angiosomes of the medial superficial epigastric artery [11].
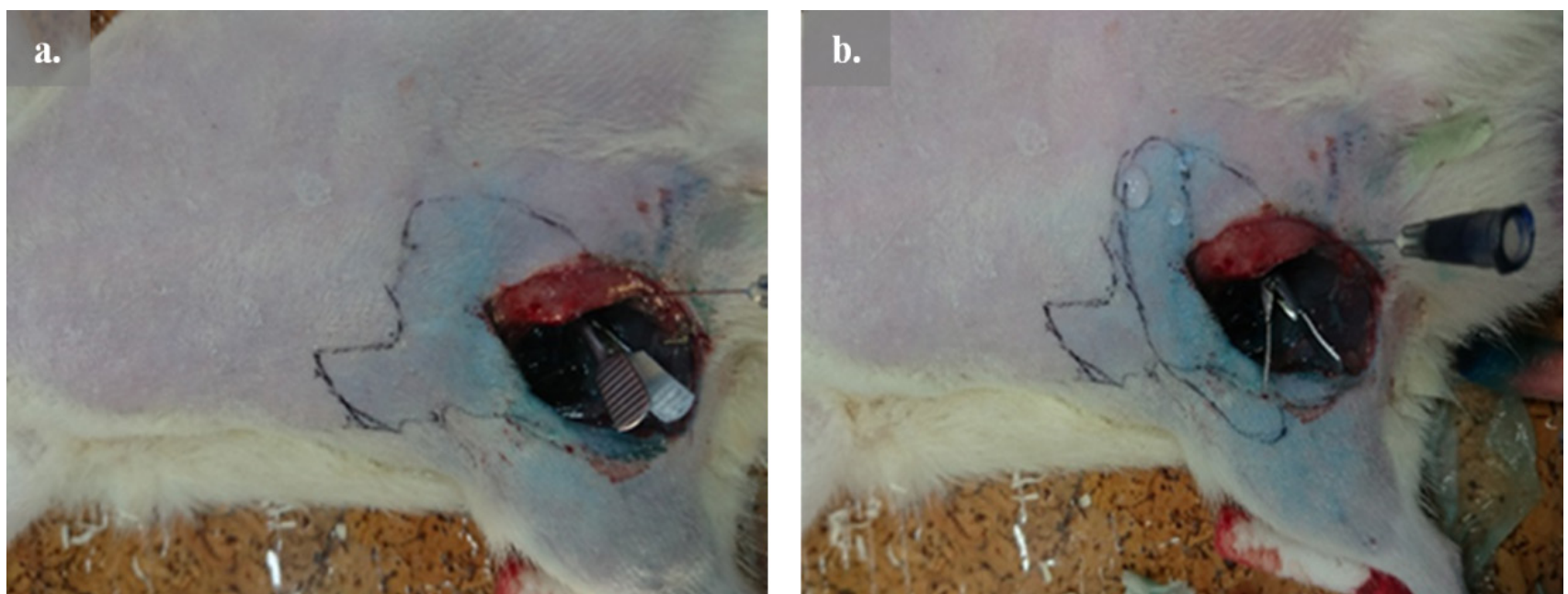

Figure 13. Functional perforasome revealed by methylene blue injection: a. Functional perforasome; b. Anatomic perforasome after choke vessels constriction. 

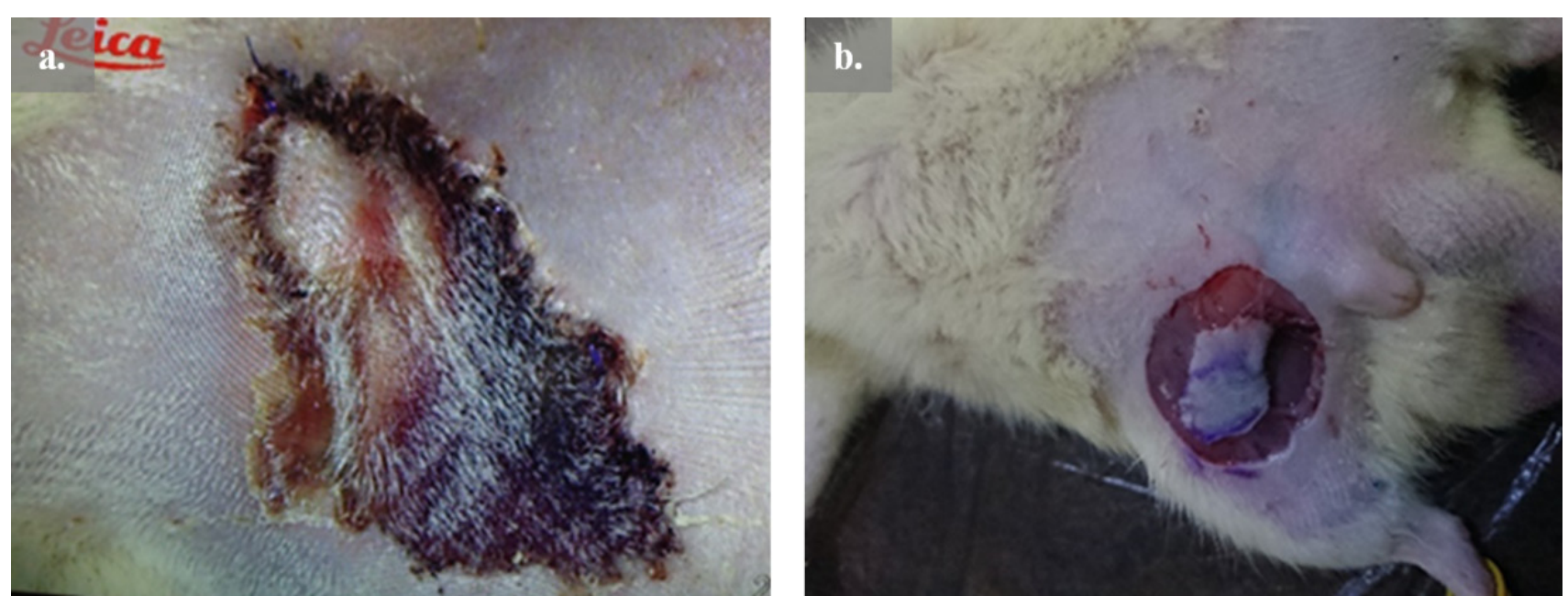

Figure 14. The flap designed wider than the functional perforasome: a. Partial flap necrosis; $\mathbf{b}$. The flap design outside of functional perforasome boundaries.

The injection of the dye needs the catheterisztion of the femoral artery and clamping of the other branches. We observed that the optimal time of ischemia for the limb was under 50 minutes, a longer time of ischemia leading to limb necrosis.

When using methylene blue, the dye injection should be continuous because 1-2 minutes of delay will lead to the highlight of other perforasomes due to its vasoconstrictor effect on nitric oxide at the level of choke vessels (Figure $13 \mathrm{a}, \mathrm{b}$ ). The effect of methylene blue on the choked vessels also acted as preconditioning factor for the flap, interrupting the vascular connection of the functional perforasome with the rest of the skin.

The accuracy of the method was demonstrated by designing the flap wider than the functional perforasome initially marked. Partial necrosis of the flap (Figure 14 a.) in the territory which exceeded the functional perforasome was registered (Figure $14 \mathrm{~b}$ ).

When the flap was raised on the functional perforasome, in the fourth day of postoperatively evolution the dye was washed out showing the establishment of a new vascular network.

\section{Discussion}

The initial dissection until the split of the two branches of superficial epigastric artery and the clamping of all arterial vessels emergent from the femoral artery not only reduces significantly the quantity of die injected but also the time of ischemia.

The quantity of dye injected is also reduced by making the dissection from the depth to the skin, thereby avoiding the sectioning of capillaries which would lead to dye leakage on the surgical field and thus leading to errors when the quantity of the dye needed is evaluated and more dye is absorbed in tissues. One of the disadvantages of this procedure is the use of the proximal branches of the superficial epigastric artery to the incision which are direct perforators thus the dosing method does not evaluate the muscular or septal perforators for whom distal dissection of lateral branch of superficial epigastric artery is needed.

If the dye is injected before the dissection, the identification and isolation of the epigastric superficial artery is easier, but the quantity of dye absorbed by the tissue increases and the accurate identification of one perforasome may be compromised by injecting more perforators at the same time. Also, this option extends the time of ischemia which will lead to limb necrosis and autophagy. Although methylene blue reduces inflammatory factors, attenuates skeletal muscle damage and lung injury, the quantity and concentration are insufficient to preserve the limb in case of prolonged ischemia, more than 50 minutes, determining only a slower evolution of necrosis [14].

In our previous review [12] the effect of the dye on different tissues of the dye was analyzed thereby knowing already that the range of concentration used in this study is not toxic for the tissue involved, as histopathological studies demonstrated. Knowing that the dye effects are dose and concentration-dependent, a lower concentration will require the injection of a greater quantity of dye which, in case of methylene blue could highlight additional perforasome and at the same time lead to errors (Figure $14 \mathrm{a}, \mathrm{b}$ ). 


\section{Conclusions}

Intraoperative evaluation of functional perforasome is one of the best options to prevent perforator flap necrosis. It has an ideal harvesting surface definition accuracy and does not need any special devices: a safe biological concentration has good visibility with the naked eye and the dye is not expensive.

\section{References}

1. Kroll SS, Rosenfield L. Perforator-based flaps for low posterior midline defects. Plast Reconstr Surg. 1988;81:561-566.

2. Koshima I, Soeda S. Inferior epigastric artery skin flaps without abdominis muscle. Br J Plast Surg. 1989;42:645-648.

3. Hashimoto I, Abe Y, Ishida S, Kashiwagi K, Mineda K, Yamashita Y, et al. Development of Skin Flaps for Reconstructive Surgery: Random Pattern Flap to Perforator Flap. J Med Invest. 2020;63:159-162.

4. Hallock GG. The complete nomenclature for combined perforator flaps. Plast Reconstr Surg. 2011;127:1720-1729.

5. Lecours C, Saint-Cyr M, Wong C, Bernier C, Mailhot E, Tardif M, et al. Freestyle pedicle perforator flaps: clinical results and vascular anatomy. Plast Reconstr Surg. 2010;126:1589-1603.

6. Chih-Hsun L, Ma H. Use of Perforator-Based Fasciocutaneous Flaps for Pressure Sore Reconstruction: Single-PerforatorBased Versus Multiple-Perforator-Based Flaps. Aesthetic Plast Surg. 2016;40:540-548.
7. Leung R, Chae MP, Tobin V, Hunter-Smith DJ, Rozen WM. In-vivo quantitative mapping of the perforasomes of deep inferior epigastric artery perforators. Plast Reconstr Surg Glob Open. 2018;6:e1960.

8. Rozen WM, Leung R, Chae MP, Hunter-Smith DJ. Imaging of perforasome territories: the evolution of techniques. Australas J Plast Surg. 2018;1:65-73.

9. Saint-Cyr M, Wong C, Schaverien M, Mojallal A, Rohrich RJ. The perforasome theory: vascular anatomy and clinical implications. Plast Reconstr Surg. 2009;124:1529-1544.

10. Zhuang Y, Hu S, Wu D, Tang M, Xu DC. A novel in vivo technique for observations of choke vessels in a rat skin flap model. Plast Reconstr Surg. 2012;130:308-317.

11. Casal D, Pais D, Iria I, Videira PA, Mota-Silva E, Alves S, et al. Blood Supply to the Integument of the Abdomen of the Rat: A Surgical Perspective. Plast Reconstr Surg Glob Open. 2017;5:e1454

12. Nedu ME, Tertis M, Cristea C, Georgescu AV. Comparative study regarding the properties of methylene blue and proflavine and their optimal concentrations for in vitro and in vivo applications. Diagnostics (Basel). 2020;10:223.

13. Casal D, Pais D, Iria I, Videira PA, Mota-Silva E, Alves S, et al. Blood Supply to the Integument of the Abdomen of the Rat: A Surgical Perspective. Plast Reconstr Surg Glob Open. 2017;5:e1454.

14. Wang L, Chen B, Lin B, Ye Y, Bao C, Zhao X, et al. Methylene blue attenuates lung injury induced by hindlimb ischemia reperfusion in rats. Mediators Inflamm. 2018;2018:2508620. 